\title{
Applications of Artificial Intelligence in the Screening of Glaucoma in China
}

\author{
Yue Zhang, Ningli Wang and Hanruo Liu* \\ Beijing Tongren Eye Center, Beijing Key Laboratory of Ophthalmology and Visual Sciences, Beijing Tongren Hospital, Capital \\ Medical University, China
}

*Corresponding author: Hanruo Liu, Beijing Tongren Eye Center, Beijing Key Laboratory of Ophthalmology and Visual Sciences, Beijing Tongren Hospital, Capital Medical University, China

ARTICLE INFO

Received: 蔧 April 09, 2020

Published: 慧 April 24, 2020

Citation: Yue Zhang, Ningli Wang, Hanruo Liu. Applications of Artificial Intelligence in the Screening of Glaucoma in China. Biomed J Sci \& Tech Res 27(2)2020. BJSTR. MS.ID.004476.

\begin{abstract}
Glaucoma is a main cause of irreversible blindness, with the difficulty in identifying a large number of undiagnosed patients at early stages. Artificial intelligence (AI) has fostered new breakthroughs in automated screening for glaucoma. Recent years, large amounts of studies have been conducted to employ AI in screening and diagnosis of glaucoma in China, which demonstrates great performance in the procedure. It is expected to revolutionize the current management process of diseases and prevent visual ailments, while practical challenges should also be taken into account.
\end{abstract}

Keywords: Artificial Intelligence; Glaucoma

Abbreviations: AI: Artificial Intelligence

\section{Introduction}

Glaucoma is a main cause of irreversible blindness, which is characterized by structural changes in the optic nerve head, death of retinal ganglion cell and loss of visual acuity, affecting estimated 64.3 million patients aged from 40 to 80 years worldwide [1,2]. Glaucoma meets most of the criteria for screening. First, most cases of chronic glaucoma may be asymptomatic at early stages, and the majority ofirre versible loss of vision can be prevented by timely diagnosis and treatment [3]. Next, the screening test (retinal photography) is simple, safe and validated. Last but not least, glaucoma has become an important public health problem. Screening for glaucoma has been recommended by many countries and international community's [4]. Artificial intelligence (AI) has fostered new breakthroughs in automated screening and diagnosis for glaucoma. Implementation of AI in ophthalmic images, including fund us photographs and visual fields, has been proved to be featured by high accuracy, sensitivity, and specificity of over $90 \%$ [5]. It is believed that AI may serve as a potential alternative to ophthalmologists and trained human graders in the screening of glaucoma.

In China, the prevalence of glaucoma is about $2.6 \%$ in people over 40 years old and increases with age, whose blindness rate is $30 \%$ [6]. Different from other countries, Features of vast population, uneven quality of health care and high proportion of open-angle glaucoma increase the difficulty of screening in China. A considerable amount of research has been conducted to employ AI in the screening of glaucoma. In 2018, Li et al. [7] Developed a deep learning system for detecting glaucomatous optic neuropathy which outperformed ophthalmologists. In 2019, Beijing Tongren Hospital and Beijing Institute of Ophthalmology developed a deep learning algorithm for automatically detecting glaucomatous optic nerve changes in fundus images [8]. It showed the sensitivity of $96.1 \%$ and specificity of $97.1 \%$ by assessment, and a prediction visualization test was performed to better understand the decisionmaking process [9]. Moreover, Beijing Tongren Hospital also developed the first screening product for glaucoma in China by cooperating with Tencent Medical Health, with an accuracy rate of more than $95 \%$. This product has been launched on linesince June 2019.

Strengths of these studies are clear. Integrating AI well into glaucoma practices is expected to revolutionize the current management process of diseases and prevent visual ailments, thereby alleviating various social burdens in China. However, several challenges exist in adoption of such systems for clinical translation and utility in glaucoma screening programs. First, there are limitations in present studies, which have been acknowledged 
by many researchers. For example, it remains unknown that what the exact mechanism is for AI to evaluate features and make predictions. Moreover, fundus images alone is not aguaranteed sign of glaucoma and the evaluation of glaucomatous optic nerve changes is highly subjective, especially in diagnosing glaucoma in earlier stages. Another limitation is that most systems lack inability to detect other important eye conditions, especially high myopia, which is the most common reason for false-negative and falsepositive in both human graders and AI. Thus a more developed and integrated system is necessary to improve screening applications in China.

The second important challenge to use $\mathrm{AI}$ is that the application and validation of these advanced methods in thereal-world screening setting need additional investigations to bolster its support. In consideration of the non uniform distribution of population and differences in prevalence around China, it is important to compare the performance of AI in populations of different prevalence of glaucoma. In addition, shooting quality and diagnosis ability are inconsistent even in higher-level hospitals across China, especially in economically underdeveloped regions. It will be the first and immediate barrier to broad implementation of AI applications in China. The third challenge is about how to fit AI well into the health care system, and whether this procedure has cost-effectiveness. At present, most scenes accepting applications are hospitals with relevant backward medical resources and primary care clinics. However, to what extent should anophthalmologist or optometrist trust the AI outcome? In low-resource settings, like western regions, where professional ophthalmologists are in deeply need, what should we suggest the individuals with suspected glaucoma to get further confirmation? A fundamental approach to solve the problem might be to balance the distribution of medical resources around the country.

The forth challenge is about how to avoid doctor-patient conflicts in primary medical sites. According to the Ministry of Health of the People's Republic of China (China Annual of Sanitation, 2017), over the last twenty years, conflicts between patients, families and doctors have been escalatingin China, with growing $22.9 \%$ annually. Patients show a lack of trust in professional authorities of primary health care providers, and hesitanceto give full control of their bodies to doctors. Accordingly, it is welcomed to integrate $\mathrm{AI}$ into the health care systemtimely and effectively, while the practical challenges should also be taken into account. Further benefits will inevitably come from the use of AI with digital images and multiple other orthogonal datasets, such as cardiovascular and genomic data, which will enhance the value of data utilization for the health care system.

\section{Conflict of Interest}

No potential conflict of interest relevant to this article was reported.

\section{Acknowledgment}

This research was supported by National Natural Science Fund Projects of China (81700813);Beijing Municipal Administration of Hospitals' Youth Programme (QML20180205);The priming scientific research foundation for the junior researcher in Beijing Tongren Hospital, Capital Medical University (2016-YJJZZL-021); Beijing Tongren Hospital Top Talent Training Program, Medical Synergy Science and Technology Innovation Research (Z181100001918035).

\section{References}

1. Weinreb RN, Aung T, Medeiros FA (2014) The path physiology and treatment of glaucoma: A review. JAMA 311(18): 1901-1911.

2. Stevens GA, White RA, Flaxman SR, Price H, Jonas JB, et al. (2013) Global prevalence of vision impairment and blindness: magnitude and temporal trends, 1990-2010. Ophthalmology 120(12): 2377-2384.

3. Heijl A, Bengtsson B, Oskarsdottir SE (2013) Prevalence and severity of undetected manifest glaucoma: results from the early manifest glaucoma trial screening. Ophthalmology 120(8): 1541-1545.

4. Thomas SM, Jeyaraman MM, Hodge WG, Hutnik C, Costella J, et al. (2014) The effectiveness of teleglaucoma versus in-patient examination for glaucoma screening: A systematic review and meta-analysis. PLoS One 9(12): e113779.

5. Ting DSW, Pasquale LR, Peng L, Campbell JP, Lee AY, et al. (2019) Artificial intelligence and deep learning in ophthalmology. Br J Ophthalmol 103(2): 167-175.

6. Liang YB, Friedman DS, Zhou Q, Yang X, Sun LP, et al. (2011) Prevalence of primary open angle glaucoma in a rural adult Chinese population: the Handan eye study. Invest Ophthalmol Vis Sci 52(11): 8250-8257.

7. Li Z, He Y, Keel S, Meng W, Chang RT, et al. (2018) Efficacy of a Deep Learning System for Detecting Glaucomatous Optic Neuropathy Based on Color Fundus Photographs. Ophthalmology 125(8): 1199-1206.

8. Li L, Xu M, Liu H, Li Y, Wang X, et al. (2019) A Large-scale Database and a CNN Model for Attention-based Glaucoma Detection. IEEE TRANSACTIONS ON MEDICAL IMAGING 1(2):1-11.

9. Liu H, Li L, Wormstone IM, Qiao C, Zhang C, et al. (2019) Development and Validation of a Deep Learning System to Detect Glaucomatous Optic Neuropathy Using Fundus Photographs. JAMA Ophthalmol 137(12): 1353-1360. 
ISSN: 2574-1241

DOI: 10.26717/BJSTR.2020.27.004476

Hanruo Liu. Biomed J Sci \& Tech Res

(c) (P) This work is licensed under Creative

Submission Link: https://biomedres.us/submit-manuscript.php

$\begin{array}{ll}\text { BIOMEDICAL } & \text { Assets of Publishing with us } \\ \text { RESEARCHES } & \text { - Global archiving of articles } \\ \text { - Immediate, unrestricted online access }\end{array}$

